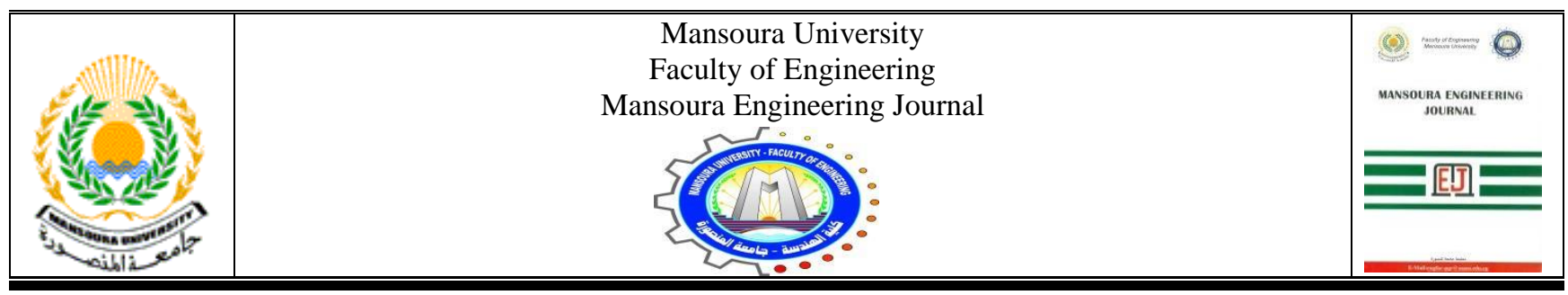

\title{
The Effect of Using the Blue Color in Interior Design for People with Intellectual Disabilities
}

\author{
I. Elaraby and Ossama A. Abdou
}

\author{
KEYWORDS: \\ mental retardation, blue \\ color, intelligence tests, \\ impact.
}

\begin{abstract}
This paper aims to study the effect of blue color on users of architectural spaces in general, and for people with intellectual disabilities in particular, with the aim of improving their mental skills. By controlling the color of the interior space and studying the effect of color on many cases through their presence in the specified space and their exposure to color. The study included 73 cases with different mental problems. We exposed 33 cases to Blue, while we left another group of $\mathbf{4 0}$ cases without exposure to it. Exposing cases to blue took two hours a day for two days a week. Intelligence tests were done for each condition, pretest (at the start of the study), and posttest (six months after the first test). The study showed that the exposure to blue color made an increase in the percentage of intelligence of cases that were exposed to blue color compared to those that were not exposed to it. Since this research aims to study the possibility of increasing the intelligence of cases with different mental problems, the aim was to study the effect of blue to increase their Intelligence quotient (IQ) compared to those who are not exposed to it. The intelligence tests that applied to different cases of people with intellectual disabilities compared to those that were not exposed to blue expected to show if there is an impact.
\end{abstract}

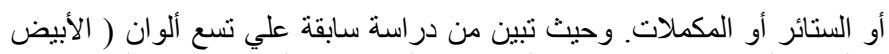

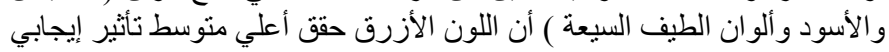

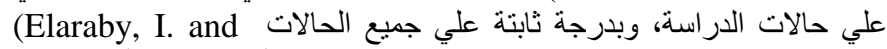
Aly, R., 2020)

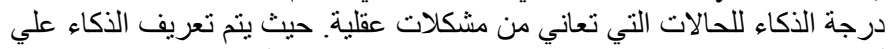

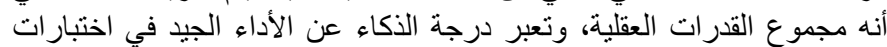

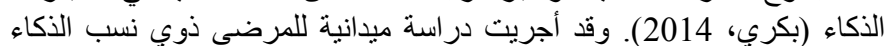

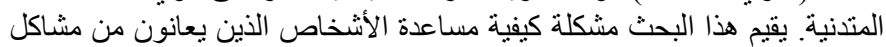
عقلية باستخدام الألوان. كان هدف البحث هو تحديد إمكانية التأثير الإيجابي علئ علي الإني

$$
\text { الأشخاص ذوي الإعاقات الذهنية. }
$$

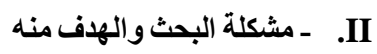

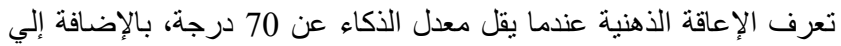

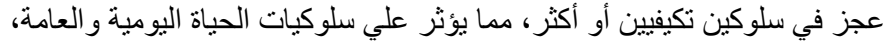

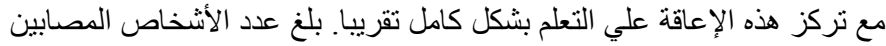
بهذه الإعاقة نحو 95 مليون شخص الإعل علي مستوي العالم في عام 2013

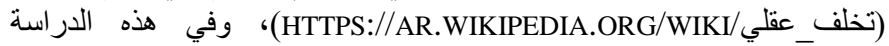
نهدف لمساعدة الأشخاص ذوي الذكاء المنخفض (أقل من 70 درجة) علي تحسين

$$
\text { درجة ذكائهم وبالتالي تحسين أدائهم السلوكي وقداع التهاء التهم التعليمية. }
$$

I I

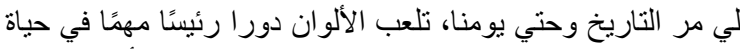

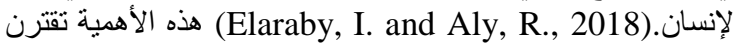

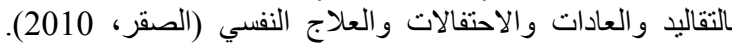

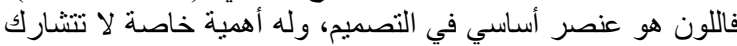

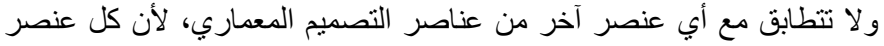

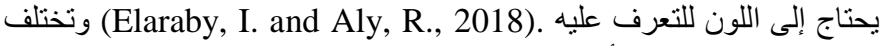
(Jung and الحساسية والاستجابة للألوان من شخص إلى آخر اختلافا كبيرا . others, 2018).

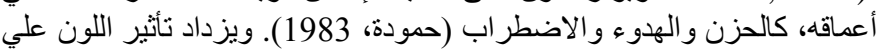

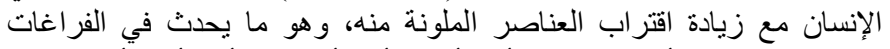

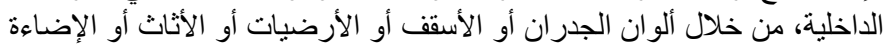

Received: (18 March, 2020) - Revised: (17 January, 2021) - Accepted: (17 February 2021)

Corresponding Author: Ibrahim Mohamed Elhady., Architecture, (email:ib_elhady@yahoo.com).

Ossama A. Abdou. is Professor of Architecture - Department of Architecture - Faculty of Engineering - Misr International University (email:(oabdou@hotmail.com). 


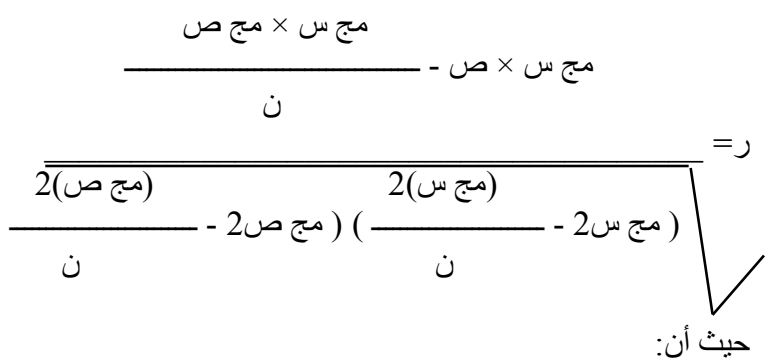

س= المتغير الأول ـ ص= الدتغير الثاني ـ ن = عدد أفراد العينة.

2-5 تعريض إحدي الدجمو عتين للون الازرق

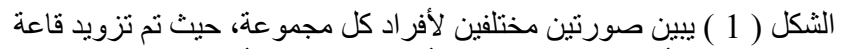

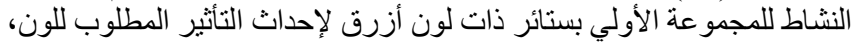

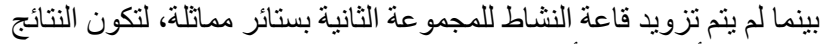

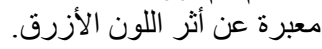

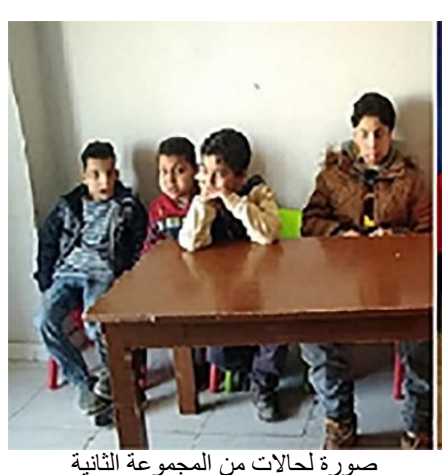

صورة لحالات من المجموعة الثانية

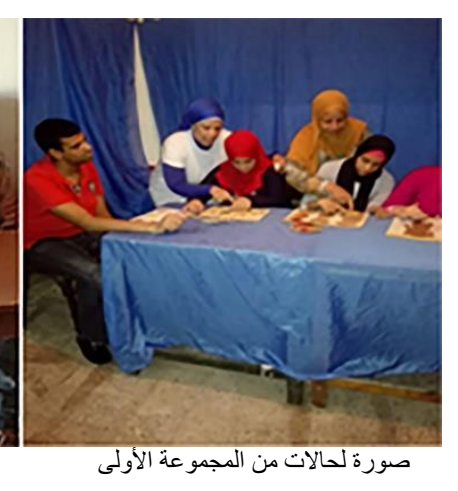

شكل رقم (1) صور لأفراد من المجمو عتين أثناء تواجدهم بمركز تتمية المهارات

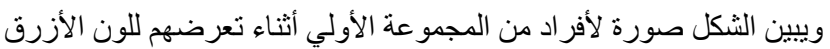

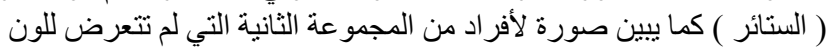

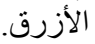

\section{VI}

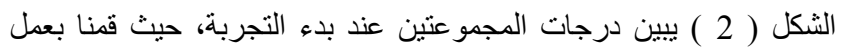

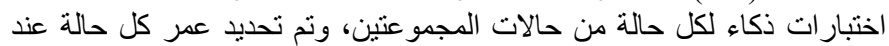

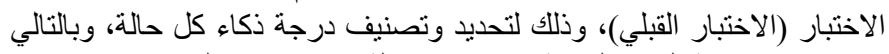
مقارنتها مع درجة الذكاء الناتجة عن الاختبار الثاني (الاختبار البعدي).

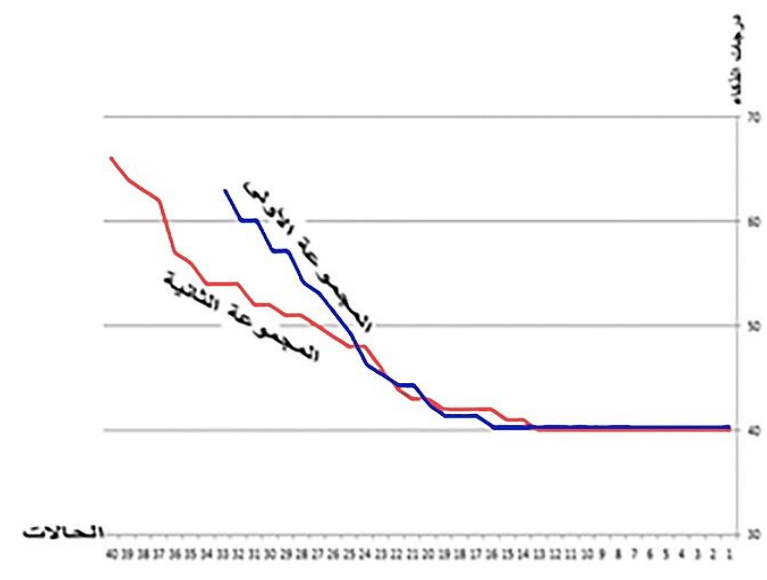

شكل رقم (2) درجات أفر اد المجمو عتين عند بدء التجربة

\section{III}

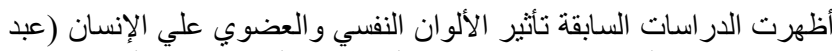

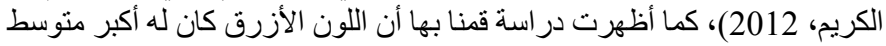

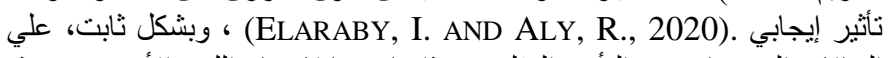
الحالات التي تعاني من التأخر العقلي، و هذا ما دفعنا لاختبار اللون الأزرق في هذه

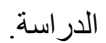

\section{IV}

اعتمدت منهجية البحث علي الخطو ات التالية

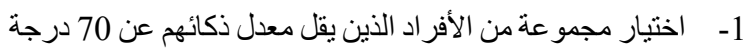

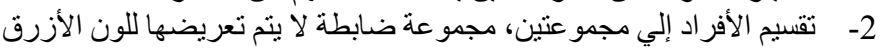

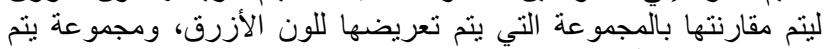

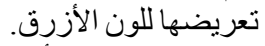
3- قياس درجات ذكاء أفر اد المجمو عتين قبل بلدء التجربة ثم إعادة القياس بعد 6

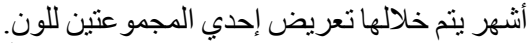
4- قياس الفرق بين الدرجات القبليّة والبعدية ليعرفة أثر اللون.

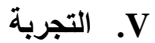

تم اختيار حالات معدل ذكائها أقل من 70 درجة ، و وتم تقسيم الحالات إلي

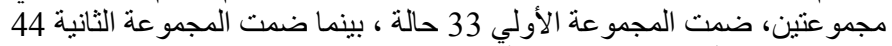

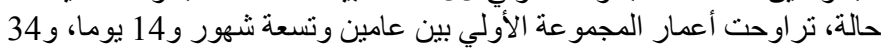

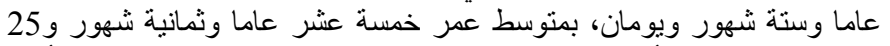

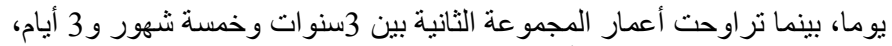

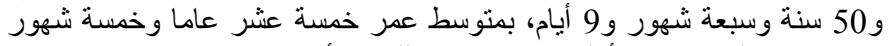

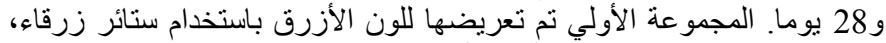

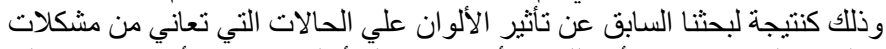

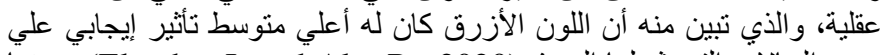

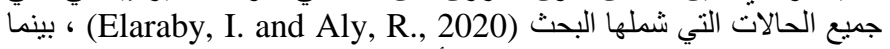

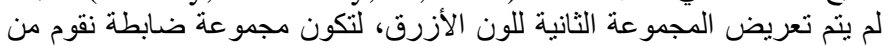

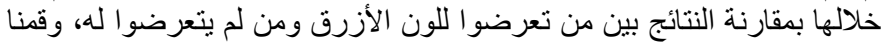

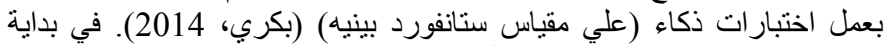

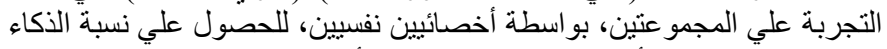
الكلي علي الكقياس ثم أعدنا الاختبار ات بعد ستة أنثهر علي المجمو عتين، وحصلنا علي النتائج التالية

1-5 الدلات الإحصائية لتأكيد مصداقية النتائج قمنا بعمل اختبارين إحصائيين 1-1-5 اختبار (ت ) للكشف عن دلالة الفروق الإحصائية. تم حساب قيمة ( ت ) للمجمو عتين حيث : (د) الفرق بين الدرجتين، القبلية و البعدية. (د1) القيمة المتوسطة للفرق

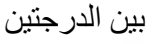

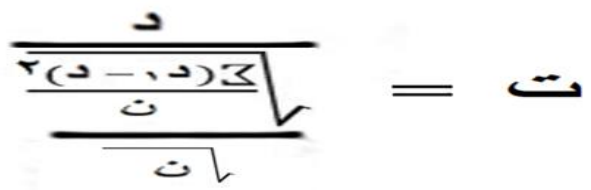

وبحساب قيمة ت للمجموعة الأولي كان الناتج 0.295 وهو أقو أقل من القيمة

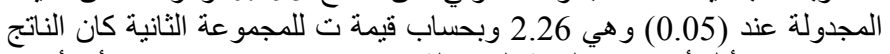

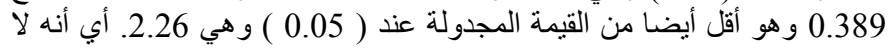
توجد فروق إحصائية ذات دلالة في المجمو عتين.

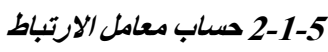

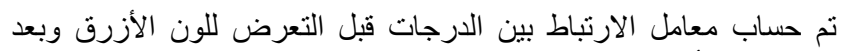

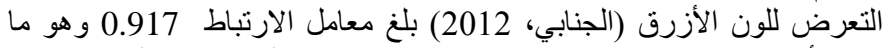

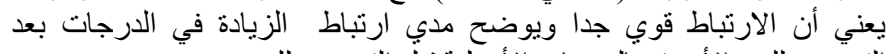
التعرض للون الأزرق بالدرجات الأصلية قبل التعرض آرباط للون. 
يتضح من الشكل أن الزيادات الكبيرة كانت ذات علاقة بالعمر الأقل حيث

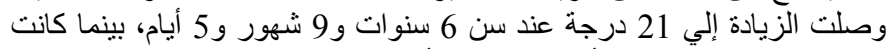
الزيادات بقيمة صفر في الأعمار الكبيرة، أما القيم السالبة فارتبطت بنس بن المن المر اهقة

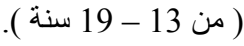

6- 3 علاقة الزيادة في نسبة الذكاء بالنوع

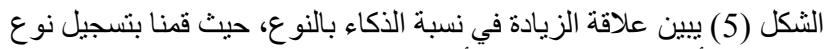

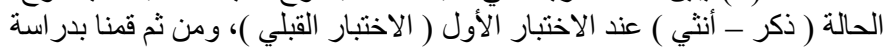

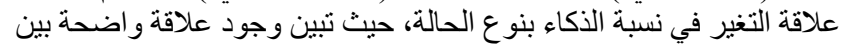
التغير في نسبة الذكاء ونوع النغير فاء لنوع كما في الثكلة.

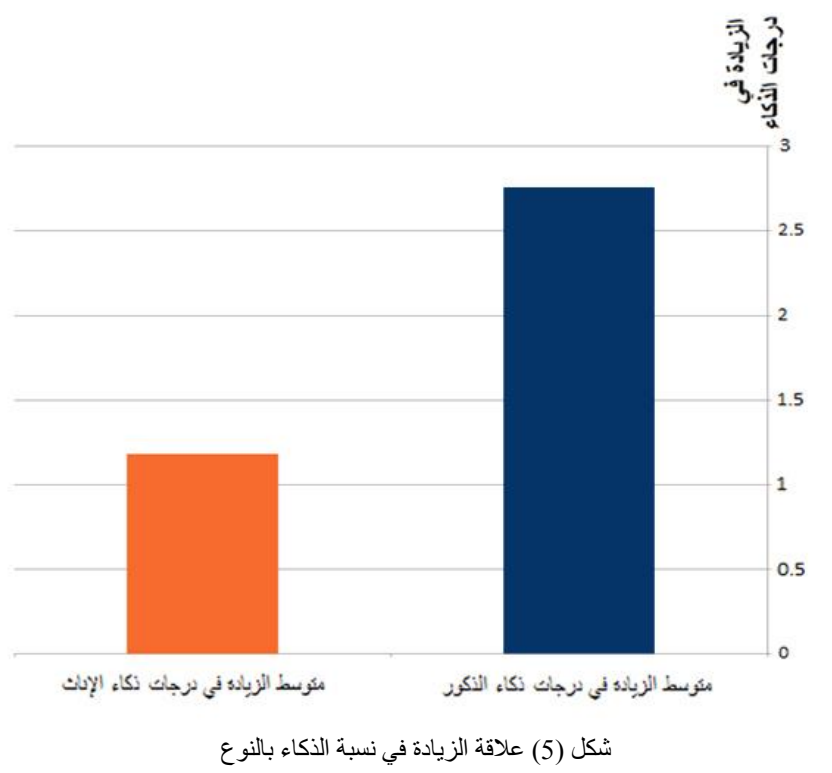

يتضح من الثكل أن متوسط الزيادة في نسبة الذكاء للذكور بلغ أكثر من الزباء

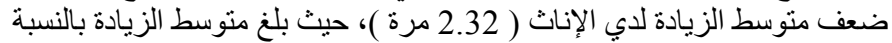

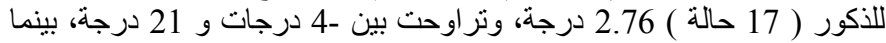

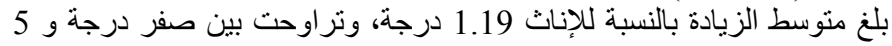
درجات.

\section{6- 4 علاقة الزيادة في نسبة الذكاء بالدرجات الأصلية}

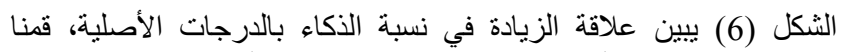

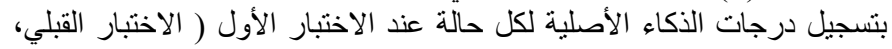

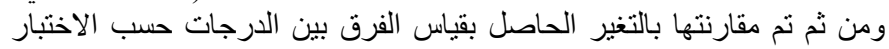
الثاني ( الاختبار القبلي ) والدرجات الناتيات الناتجة من الاختبار الثاني (الاختبار البعدي)

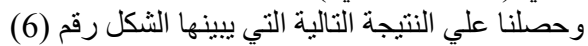

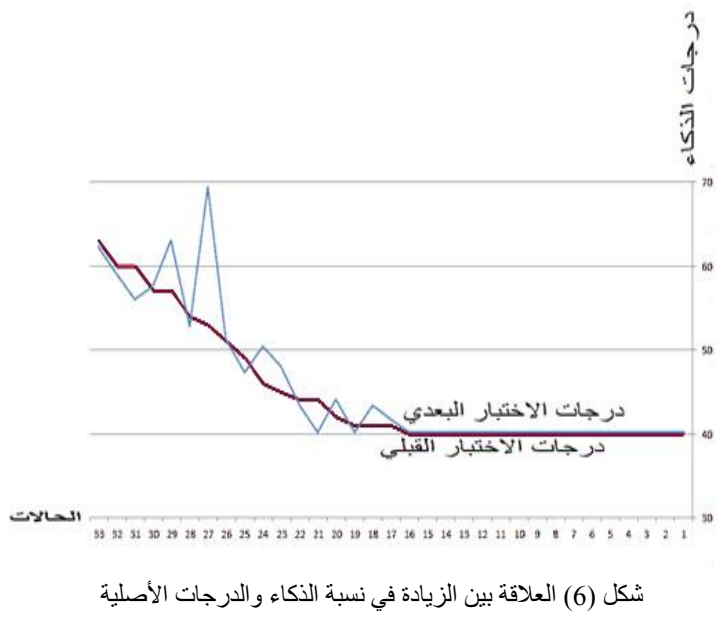

ويبين الثكل ( 2 ) أن درجات الذكاء للمجموعة الأولي ( باللون الأزرق )

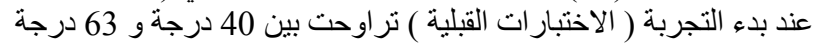

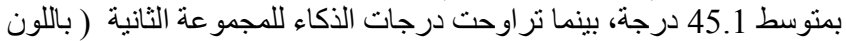
الأحمر ) عند بدء التجربة ( الاختبار ات القبلية ) بين 46. بمتوسط 46.9 درجة، كما يبين التقارب بين درجات الإن المجو عتين.

$$
\text { 6-1 الزيادة في نسبة الذكاء وفقا للاختبار الثاني }
$$

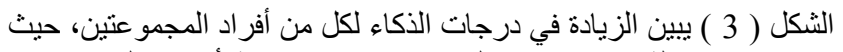

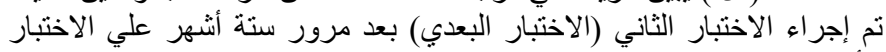

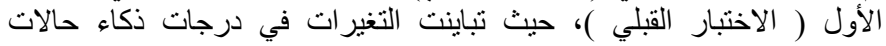

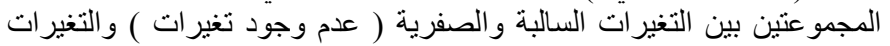

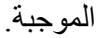

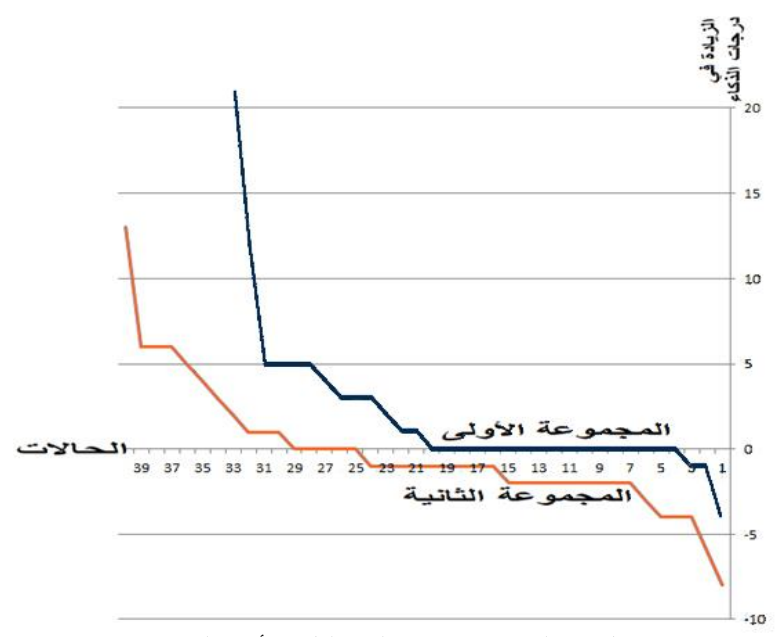

شكل (3) الزيادة في درجات الذكاء لكل من أفراد المجمو عتين

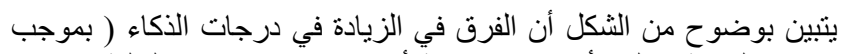

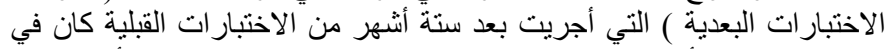

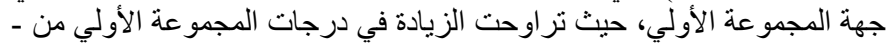

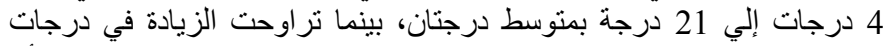

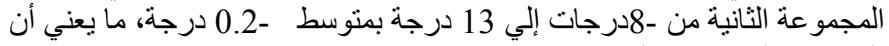
الفرق في الزيادة بين المجمو عتين يساوي 2.2 درجة.

$$
\text { 2-6 الزيادة في نسبة الذكاء وعلاقتها بالعدر }
$$

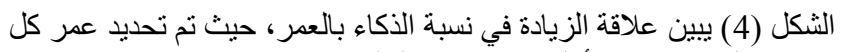

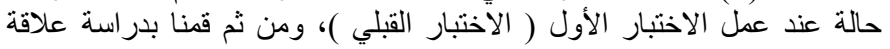
العمر بالتغير في نسبة الذكاء، مقارنة بالاختبار الثاني (الاختبار الاخبار البعدي)، وحصلنا علي النتائج التالية

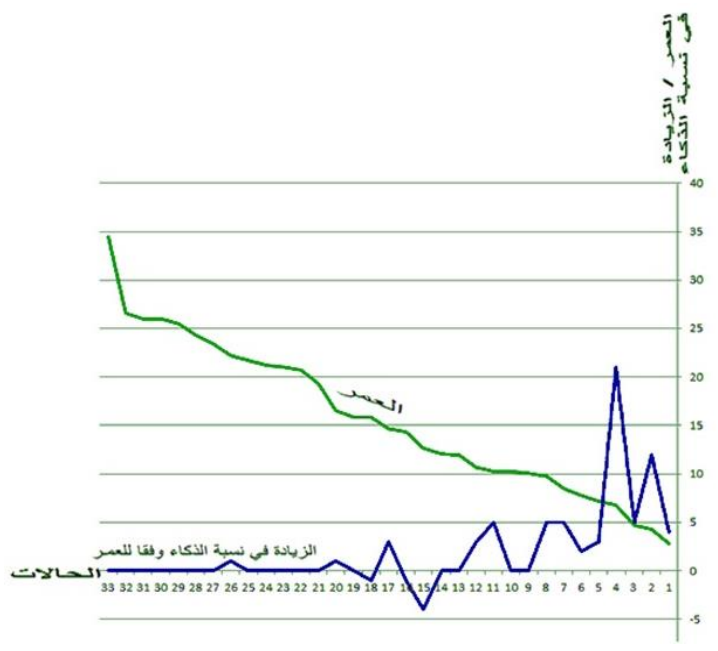

شكل ( 4 ) علاقة الزيادة في نسبة الذكاء بالعمر 
بدء التجربة، ثم بعد ستة أشهر، ومن ثم تم احتساب منوسط الفرق في الحالتين.

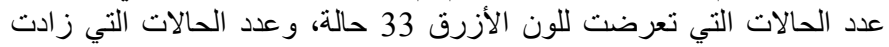

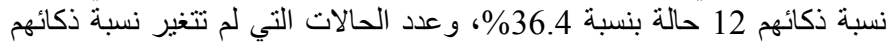

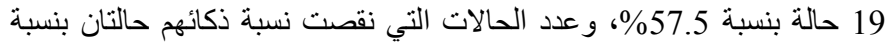

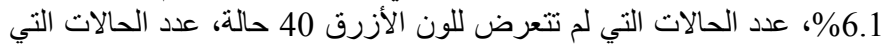

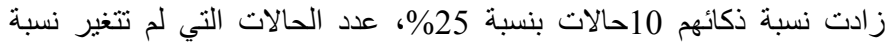

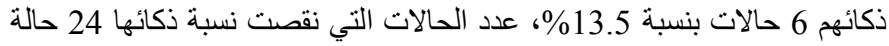

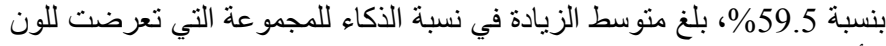

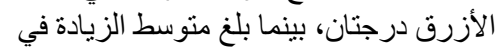

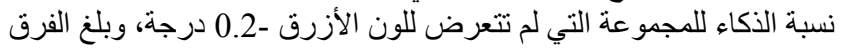

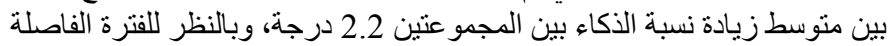

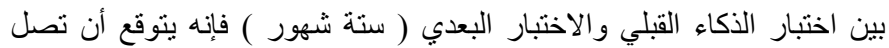

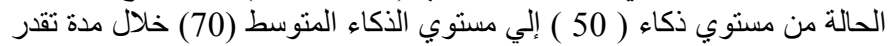
في المتوسط بأقل من خمس سنو ذاء (

\section{IX}

1- الاهتمام بلون الحيز الفراغي، واستخدام اللون الأزرق خاصة مع حالات التأخر العقلي.

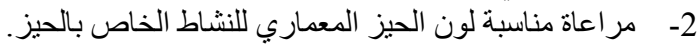

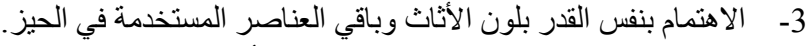

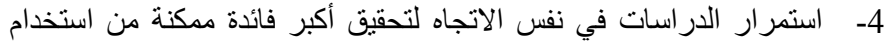

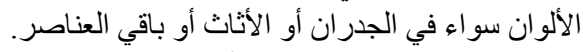

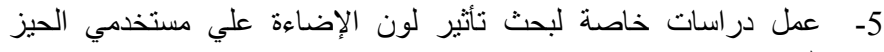
المعماري.

6- مراعاة الفروق الفردية عند تصميم حيز للاستخدام الفردي ( يفضل عمل

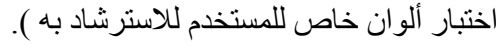

\section{المراجع العربية}

[1] [ الصقر ، إياد محم. (2010). فلسفة الألوان. عمان، الأردن: الثركة الألهلية للنشر والتوزيع.

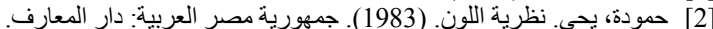

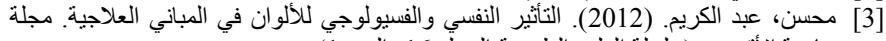
جامعة الأقصى. (سلسلة العلوم الطبيعية،المجلد 16، الإلفئ العدد 1).

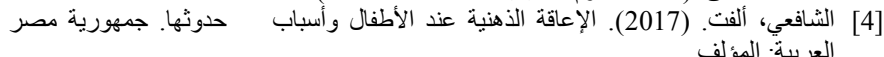

[5] ياسين، ربا محمود. (2014). أثر إدراك الألوان علي عملية الاسترجاع. (رسالة ماجيستير، كلية

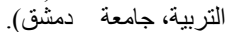

[6] جبريل، توفيق عبد الرحمن توفيق. (2013). أثر اللون في الفراغات الداخلية على النشاط

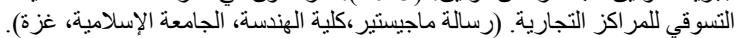

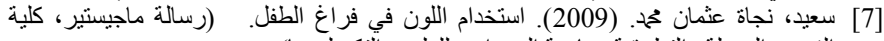

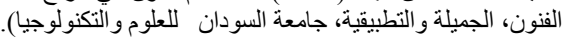

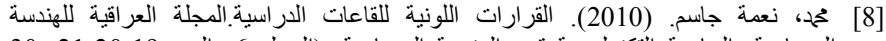

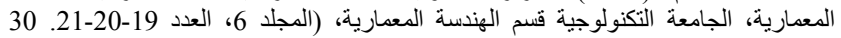
نو فمبر).

[9] بكري، سامية. (2014). الصور المختصرة لمقياس ستانفورد - بينيه، جمهورية مصر العربية: عالم الكتب.

[10] الجنابي، سلمان عكاب سرحان. (2012). محاضرة، قسم الدراسات العليا، كلية التربية الرياضية، جامعة ذي قار، الجمهورية العر اقية.

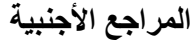

[11] Elaraby, I. and Aly, R. (2018). Power of Color. Saarbrücker, Latvia: LAMBERT, Academic Publishing.

[12] Jung, I., Elaraby, I., Griber, Y., Hanenburg, J., Arbab, Sh., Yoshimurae, K., Ulusoy, B., Johansson, S., Samir, E. (2018, September). Color Associations Different Cultures. AIC Conference, Lisbon, Portugal.

[13] Elaraby, I., and. Mohsen, R. (2013, October). Natural Colors and its Connection to Humans, AIC Conference, Newcastle, UK

[14] Darius, A. (2011). Color Inspiration. Michigan, USA: North Light.

[15] Harleman, M. (2007). Daylight Influence on Color Design. (PhD, Royal Institute of Technology, Stockholm, Sweden).

[16] Harald, A. (2012, September). Color and Light Concepts and Confusions. AIC, Taipei, Taiwan.

[17] Shahenda M., Magda, H. and Nagwa H. (2015). Designing for Attention Deficit Hyperactivity Disorder. (Master, Cairo University).

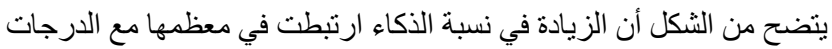

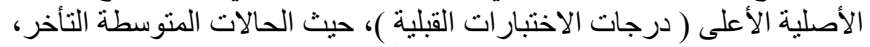
بينما لم تسجل زيادات في الحالات شديدة التأخر.

\section{.VII}
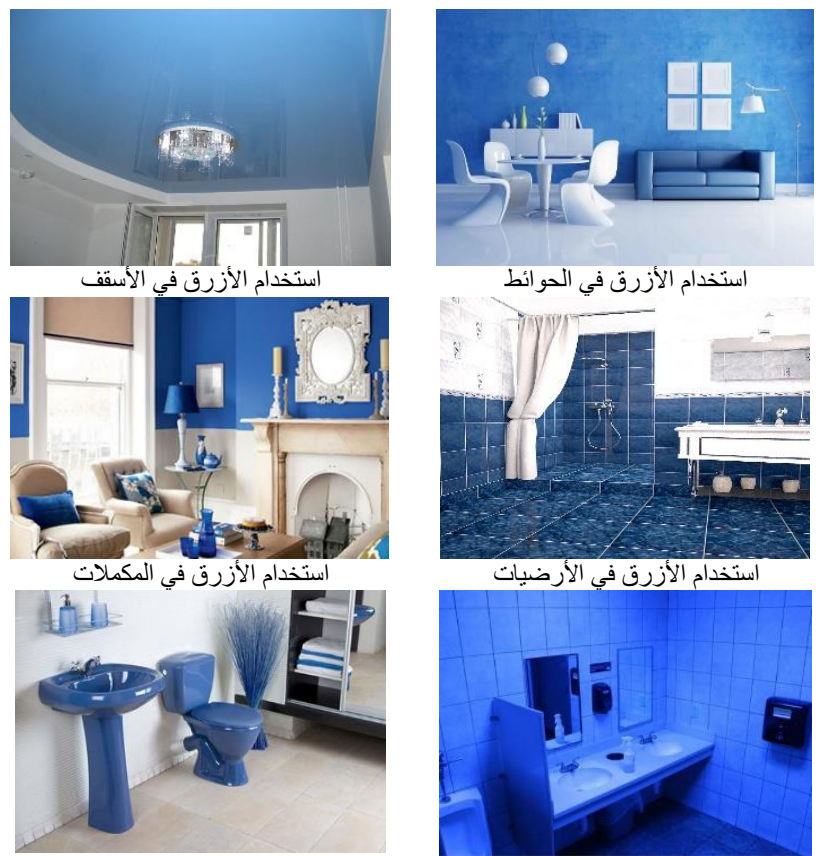

استخدام الازرق في الاررضيات
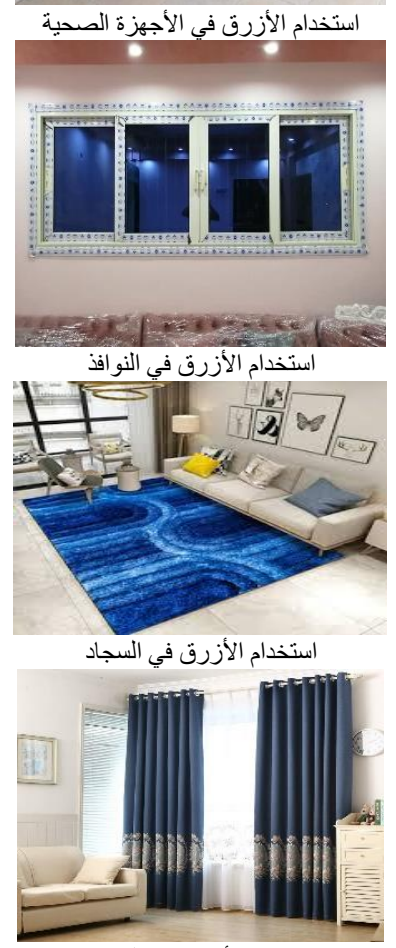

استخدام الأزرق في الستائر

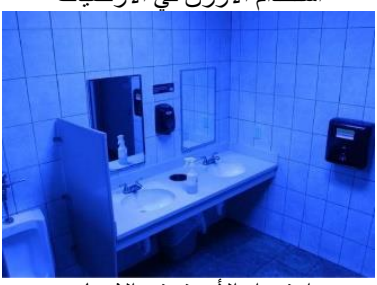

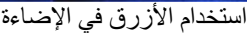

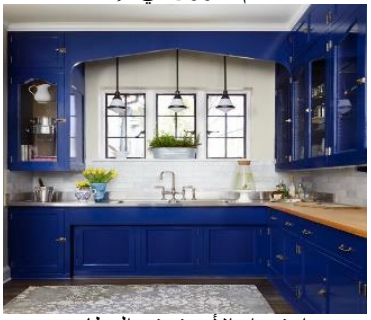

استخدام الازرق في المطابخ

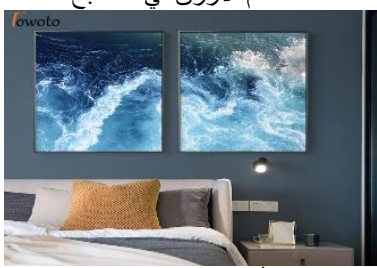

استخدام الأزرق في اللوحات الجدارية

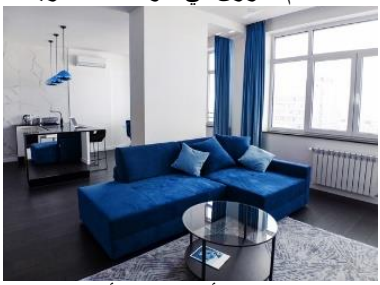

استخدام الأزرق في الأثاث

\section{-VIII}

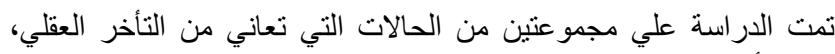

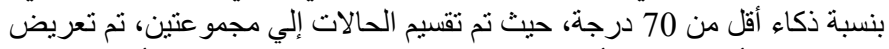

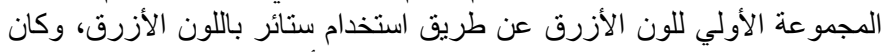

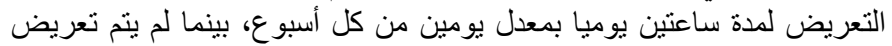

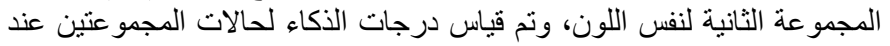


علي العديد من الحالات عن طريق تواجدها في الفراغ المحدد وتعرضها للون. شملت

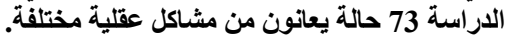

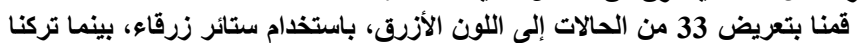

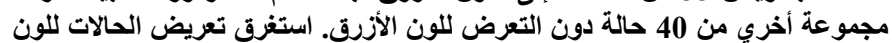

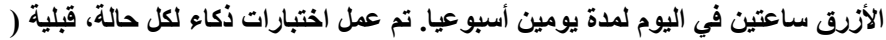

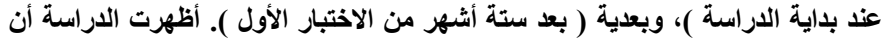

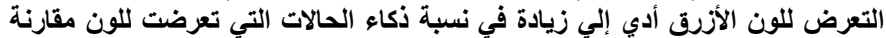

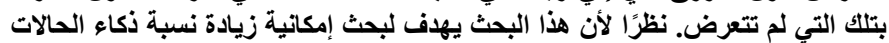

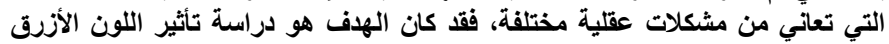

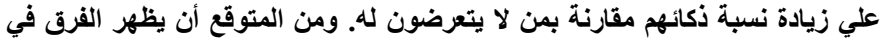

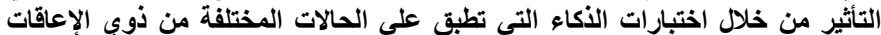
الذهنية.
[18] https://ar.wikipedia.org/wiki/\%D8\%AA\%D8\%AE\%D9\%84\%D9\%81_\% D8\%B9\%D9\%82\%D9\%84\%D9\%8A

[19]Elaraby, I., and. Aly, R. (2020, Ap). Study of the Effect of Colors Change on Children with Mental Problems. Journal of Engineering and Applied Science, Vol. 67, No. 2, APR. 2020, PP. 411-426, Faculty of Engineering, Cairo University.

\section{Title Arabic:}

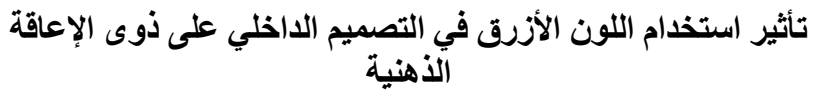

\section{Arabic Abstract:}

-تهدف هذه الورقة البحثية إلى دراسة تأثير اللون الأزرق على مستخدمي المساحات

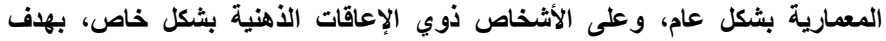
تحسين قدراتهم الذهنية. عن طريق التحكم في لون الحيز (الفراغ) ودراسة تأثير التئي اللون 